\title{
Complete genome sequences of novel S-deletion variants of porcine epidemic diarrhea virus identified from a recurrent outbreak on Jeju Island, South Korea
}

\author{
Guehwan Jang ${ }^{1} \cdot$ Jonghyun Park ${ }^{1}$. Changhee Lee ${ }^{1}[$
}

Received: 28 February 2019 / Accepted: 28 June 2019 / Published online: 26 July 2019

(c) Springer-Verlag GmbH Austria, part of Springer Nature 2019

\begin{abstract}
Porcine epidemic diarrhea virus (PEDV) is a deadly epizootic swine coronavirus that is of importance to the world pork industry. Since the re-emergence of the virulent genotype $2 b(\mathrm{G} 2 b)$ in 2014, Jeju Island in South Korea has faced periodic outbreaks, leading to the occurrence of endemics in provincial herds. In this study, we examined the complete genome sequences and molecular characteristics of novel G2b PEDV variants with a two-amino-acid deletion in the neutralizing epitope of the spike (S) gene, which were concurrently identified on a re-infected farm and its neighboring farm on Jeju Island. Whole-genome sequencing of the Jeju S-DEL isolates KNU-1829 and KNU-1830 revealed the presence of a continuous 9-nucleotide deletion within the nonstructural protein coding region. Their genomes were 28,023 nucleotides in length, 15 nucleotides shorter than those of the classical G2b PEDV strains. The two S-DEL isolates had 96.4-99.2\% and 98.3-99.7\% identity at the S-gene and full-genome level, respectively, to other global G2b PEDV strains. Genetic and antigenic analyses indicated that the S-DEL isolates are most closely related to the primary strain identified from the initial exposure at the same farm, but the virus appears to undergo continuous evolution, possibly leading to antigenic drift under recurrent or endemic pressure. This study provides important information about the antigenic diversity of PEDV circulating in the endemic areas, which arises from continuous non-lethal mutations to ensure viral fitness in the host environment.
\end{abstract}

Porcine epidemic diarrhea virus (PEDV) is a highly contagious, fatal enteric virus of pigs that belongs to the genus Alphacoronavirus in the family Coronaviridae of the order Nidovirales. Since the 2013-2014 PEDV pandemics that ravaged the American and Asian pig-raising countries, the virus has gained global attention as an emerging or reemerging viral pathogen that threatens the swine industry worldwide [6]. Outbreaks in 2013-2014 caused the death

Handling Editor: Sheela Ramamoorthy.

Electronic supplementary material The online version of this article (https://doi.org/10.1007/s00705-019-04360-4) contains supplementary material, which is available to authorized users.

Guehwan Jang and Jonghyun Park contributed equally to this work and share co-first authorship.

Changhee Lee

changhee@knu.ac.kr

1 Animal Virology Laboratory, School of Life Sciences, BK21 Plus KNU Creative BioResearch Group, Kyungpook National University, Daegu 41566, Republic of Korea of an estimated one million newborn piglets in South Korea $[6,9,10]$. Although Jeju Province had maintained a PEDVfree status for a decade, this island, located $80 \mathrm{~km}$ away from mainland South Korea at its closest point, could not prevent the spread of PEDV to provincial herds nationwide [9]. Genetic and epidemiological investigations revealed a direct incursion of the pandemic genogroup $2 b$ (G2b) PEDV from the mainland, from unknown sources [9]. PEDV has since scourged the provincial pork business and has now become endemic in Jeju pig herds $[9,11]$.

In early October 2018, enteric and diarrheal diseases accompanied by low-to-moderate mortality rates (30-50\%) in newborn piglets broke out almost simultaneously in two vaccinated farrow-to-finishing herds (farms A and B) in a Hallim-swine-dense region of Jeju Province. In particular, farm A experienced an acute PEDV infection in March 2018 [12]. Small-intestine specimens collected from diarrheic piglets at those farms were submitted to our laboratory for diagnosis. All of the samples tested PEDV positive by RT-PCR assays using a TGE/PED detection kit (iNtRON Biotechnology, Seongnam, South Korea). Subsequently, the complete sequence of the spike (S) gene of the Jeju isolates was 
determined as described previously [8]. Nucleotide sequence analysis showed that two strains, KNU-1829 and KNU1830, were genetically cognate, having $99.9 \%$ amino acid sequence identity and displaying 96.4-99.2\% identity at the amino acid level to G2b PEDVs previously reported on Jeju Island, mainland South Korea, and the USA (Supplementary Table S1). All of the isolates contained the genetic signature of the $\mathrm{G} 2$ field strains, $\mathrm{S}$ insertions/deletions (S INDELs), compared to the prototype CV777 strain, which represents the classical G1a subgroup (Supplementary Fig. S1) [6, 8]. Genetic analysis indicated that the isolates were most closely related to the KNU-1815 strain (GenBank accession number MK032689), which was identified on farm A in early 2018
[12]. Both the KNU-1829 and -1830 viruses were found to contain a novel two-amino-acid (RG) deletion at positions 1,370 and 1,371 in the C-terminal region of the $\mathrm{S}$ protein, named S DEL, which is not present in the genome sequences of the other G1 and G2 strains available from the GenBank database (Fig. 1). The $\mathrm{S}$ genes of the KNU-1829 and -1830 strains are therefore 4,155 nucleotides long and encode a 1,384-amino-acid protein, which is six nucleotides shorter than the $\mathrm{S}$ genes of the global $\mathrm{G} 2$ field strains.

As the major antigenic determinant of PEDV, the S glycoprotein contains at least four neutralizing epitopes [13, 15]. Sequence analysis revealed that, compared to the primary KNU-1815 strain, the newly identified recurrent
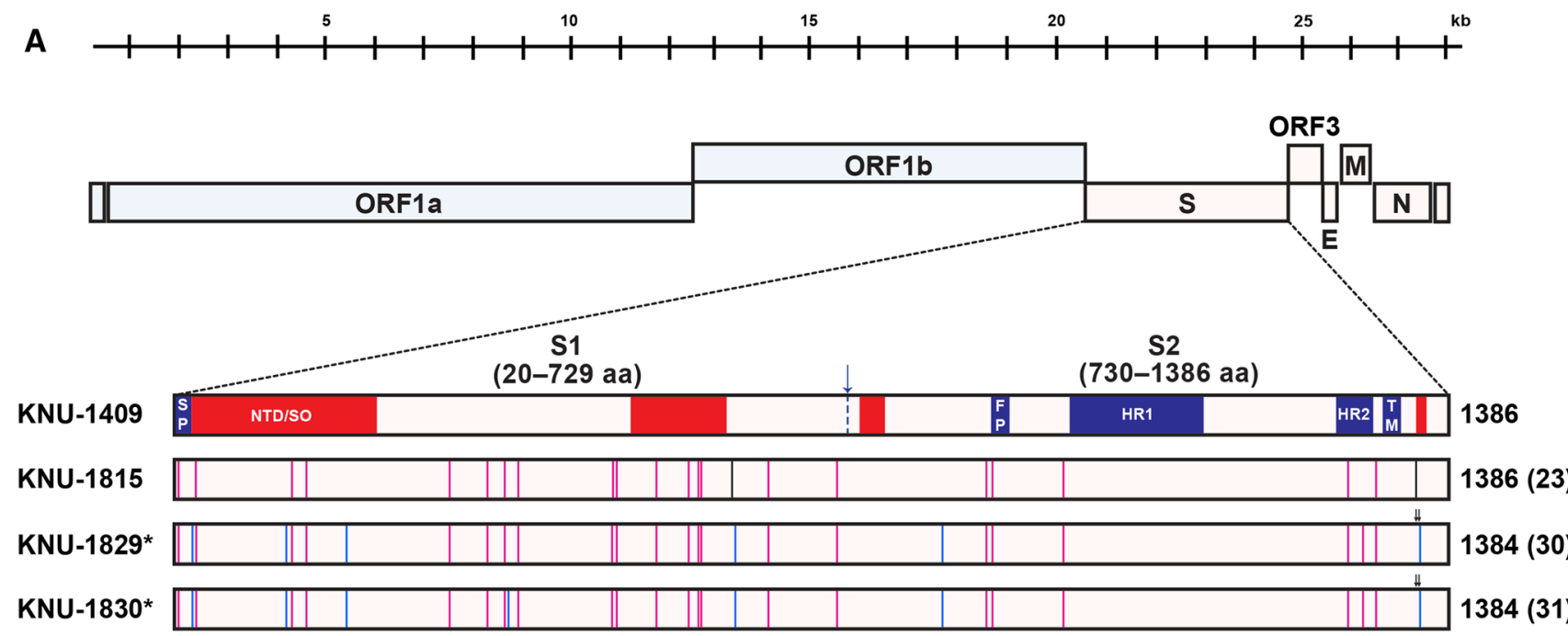

B

CV777

SM98-1

OH 851

KNU-1406-1

KNU-0801

KNU-0901

KNU-1305

KNU-141112

S DEL5/ORF3

FL2013

PC22A-P120

KNU-1815

KNU-1829*

KNU-1830 *

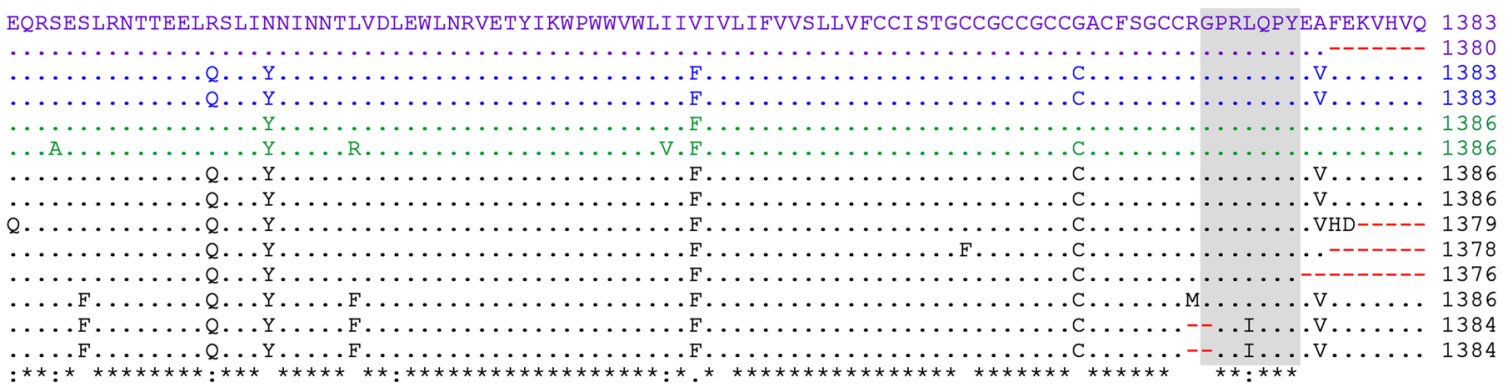

Fig. 1 Genetic variation in the PEDV S-DEL strains identified from the recurrent outbreak on Jeju Island. (A) Schematic representation of amino acid (aa) differences between the 2014 G2b Jeju re-emergent strain (KNU-1409) and 2018 isolates (KNU-1815, -1829, and -1830). The top diagram represents the organization of the PEDV genome. The $S$ protein consists of the $S 1$ and $S 2$ domains and contains a signal peptide (SP), an N-terminal hypervariable domain (NTD), a fusion peptide (FP), heptad repeat regions (HR1 and HR2), and a transmembrane domain (TM). Red areas in the diagram depicting the $\mathrm{S}$ protein represent four neutralizing epitopes (NTD/S0, aa 19-220; aa 499-600; aa 744-774; aa 1371-1377). Each vertical line represents one amino acid substitution in relation to the amino acid sequence of a reference strain. The red lines indicate common substitutions found in all isolates. The black line shows a unique mutation identified only in KNU-1815, whereas the blue lines indicate unique substitutions found in KNU-1829 and -1830. The two arrows on KNU-1829 and -1830 represent a two-amino-acid (RG) deletion. The number in parentheses on the right indicates the number of amino acid changes when compared to KNU-1409. (B) Alignment of the C-terminal amino acid sequences in the $\mathrm{S}$ proteins of the global PEDV strains. Genetic subgroups of PEDV are indicated by different colors: G1a (purple), G1b (blue), G2a (green), and G2b (black). Dashes (red color) indicate deleted sequences. The GPRLQPY epitope region at positions $1371-1377$ is indicated by a shaded gray box. The PEDV S-DEL strains KNU-1829 and -1830 identified in this study are indicated by an asterisk 
strains carry one amino acid (G) deletion and one amino acid (Leu to Ile) substitution within a previously described SD125-2 neutralizing epitope (GPRLQPY) located at positions 1371-1377 [15]. We further investigated the putative antigenicity of the $\mathrm{S}$ gene using the Bepipred linear epitope prediction tool [1]. The epitopes predicted highlighted a dissimilarity in a potential B-cell epitope, corresponding to the 1371-1377 epitope region, between the primary and S-DEL variant strains, which is suggestive of potential antigenic drift resulting from the amino acid changes (Fig. 2).

To investigate the genetic relatedness of the novel S-DEL variants to their primary strain and other global PEDV strains, the full-length genomic sequences of the KNU-1829 and -1830 strains were determined using a traditional Sanger sequencing method as described previously [10]. The KOR/ KNU-1829/2018 and KOR/KNU-1830/2018 sequence data were deposited in the GenBank database under accession numbers MK482396 and MK482397, respectively. Like the primary KNU-1815 strain, the S-DEL strains also possessed a unique nine-nucleotide deletion at genomic positions 3,408-3,416, located in open reading frame 1a, which encodes nsp3 [12]. No additional INDELs were identified throughout their entire genomes. The KNU-1829 and -1830 virus genomes were 28,023 nucleotides in length, excluding the 3 ' poly(A) tail, 15 nucleotides shorter than most $\mathrm{G} 2 \mathrm{~b}$ viruses, due to the deletions in nsp3 and S. The Jeju S-DEL isolates shared a high level of nucleotide sequence identity to each other (99.9\%) and to the other global G2b PEDV strains (98.3-99.7\%) at the genomic level (Supplementary Table S2). The highest nucleotide sequence identity was evident with respect to the primary strain, KNU-1815, indicating that virus circulation and evolution has occurred in the aforementioned farm and that there had been direct transmission to the neighboring herd. The number of nucleotide or amino acid differences and the percentage of identity between the Jeju S-DEL isolates and their primary strain KNU-1815 is summarized in Supplementary Table S3. Compared to the complete KNU-1815 genome, the KNU1829 and -1830 genomes had 61 nucleotide (99.7\% identity) and 57 nucleotide ( $99.7 \%$ identity) differences, respectively, resulting in 26 and 24 non-silent point mutations (15 in ORF $1 \mathrm{ab}, 10$ in S, and one in $\mathrm{N}$ for KNU-1829 and 12 in ORF $1 \mathrm{ab}, 11$ in $\mathrm{S}$, and one in N for KNU-1830).

Phylogenetic analysis based on the complete $\mathrm{S}$ protein clearly divided the PEDV strains into two distinct genogroup clusters, G1 and G2, which were further divided into subgroups 1a, 1b, 2a, and 2b (Supplementary Fig. S2A). The KNU-1829 and -1830 strains still belong to subgroup G2b, which clustered closely around the contemporary domestic isolates, thereby forming an independent clade within the same subgroup. Whole-genome phylogeny indicates that the novel S-DEL strains are grouped within the same cluster as the global epidemic strains (Supplementary Fig. S2B).

This is the first report describing the complete genome sequences of novel PEDV S-DEL variants on the Jeju Island, where PEDV outbreaks have occurred repeatedly since a large-scale re-emergence in 2014. The S glycoprotein is functionally divided into two domains, $\mathrm{S} 1$ and $\mathrm{S} 2$, each of which plays an independent role in coronavirus entry by mediating receptor binding and membrane fusion, respectively [5-7]. Several studies have shown that mutations, including substitutions and insertions/deletions (INDELs), in the $\mathrm{S}$ gene affect viral pathogenicity and host tropism $[3,6,14]$. A number of novel G2b variants with unique large or small S-INDELs have been reported in several countries that experienced the 2013-2014 PED

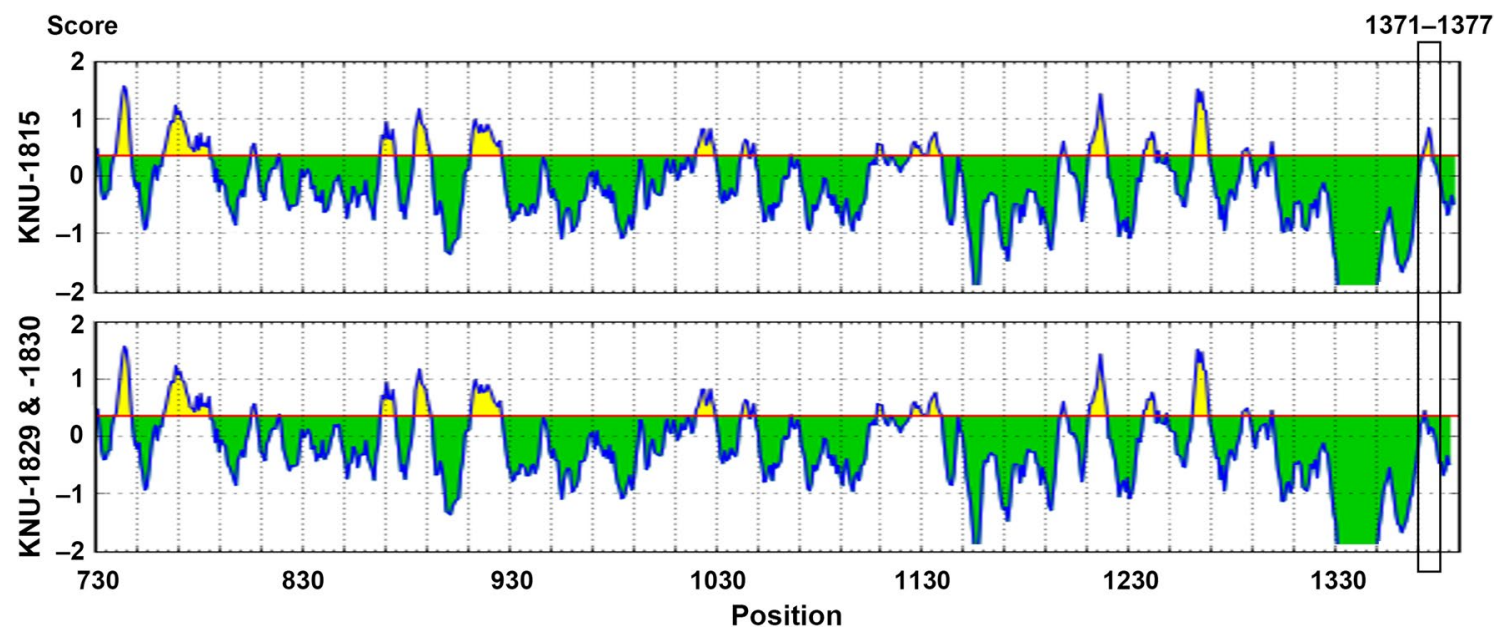

Fig. 2 Comparison of Bepipred linear epitope prediction of the S2 proteins between the primary (top) and S-DEL variant (bottom) strains. The regions with predicted epitopes in the protein are shown in yellow. The difference in the epitope region at positions 13711377 is indicated by a solid box 
pandemic and are hence possibly associated with virulence in neonatal piglets $[2,3,11,16,17]$. To investigate this hypothesis, we are currently working on isolating new PEDV variants that can grow in cell culture, the development of which will allow us to perform a challenge study. However, it is anticipated that the pathogenicity of the S-DEL strain might have been modified. Pigs infected with this novel variant experienced moderate-to-severe clinical presentation associated with PED in infected piglets; however, neonatal morbidity and mortality rates vary among litters and are lower than those reported in severe G2b outbreaks. The immune status acquired from previous natural exposures and vaccination programs may have at least contributed to the mild-to-moderate disease outcome presently observed in the field. However, despite the prior infection and vaccinations in farm $\mathrm{A}$, sow herds showed relatively low neutralizing antibody titers in their sera and colostrum (Supplementary Fig. S3). Moreover, anti-PEDV neutralizing antibodies were detectable in growing-finishing pigs, indicating the presence of circulating virus infection in this herd (Supplementary Fig. S3). This circumstance may generate immune environments that are advantageous for the survival and spread of PEDV (KNU-1815) within a farm and between farms. The virus continuously evolves under lower immune pressure to generate a new variant (KNU-1829) that may escape immune system surveillance, which in turn causes recurrences on the same farm and also new occurrences of PEDV (KNU-1830) in adjacent herds by farm-to-farm transmission via contaminated sources, including stray cats. Given its critical dual functions during cell entry, the PEDV S protein is the major target of host anti-virus antibodies, like other coronavirus S proteins [4]. A recent study revealed that the conserved S2 subunit, including its C-terminal portion, contains major antigenic determinants that strongly elicit neutralizing antibodies [15]. More importantly, the novel S-DEL recurrent variants described here have characteristic mutations within the neutralizing epitope of S compared to the primary strain, probably producing antigenic variation in the 1371-1377 epitope region. Considering these data, we hypothesize that the genetic deletions and variations in $\mathrm{S}$ that have arisen under field conditions facilitate antigenic drift, which allows the virus to evade host immune defenses, such as neutralizing antibodies, developed as a result of natural infection and vaccination. This phenomenon could weaken viral pathogenicity, resulting in less-lethal infections and leading to the establishment of an endemic state. Research involving reverse genetics is likely to provide fundamental insights into the specific roles of deletions and other mutations in PEDV pathogenesis, particularly in the putative antigenic determinants within the $\mathrm{S}$ protein. This study underscores the need to perform periodic monitoring and surveillance investigations to identify novel antigenic variants that may emerge via persistent genetic drift in enzootic situations and increase the likelihood of new PEDV outbreaks.

Acknowledgements We gratefully thank Hyong-Seok Yang and Hyekyung Yoo for providing clinical samples and critical information about the current status of PEDV on the farms.

\section{Compliance with ethical standards}

Funding This research was supported by the Basic Science Research Program through the National Research Foundation of Korea (NRF) funded by the Ministry of Education (NRF-2018R1D1A1B07040334).

Conflict of interest The authors declare that they have no conflict of interest.

Ethical approval This article does not contain any studies with animals performed by any of the authors.

\section{References}

1. Andersen PH, Nielsen M, Lund O (2006) Prediction of residues in discontinuous B-cell epitopes using protein 3D structures. Protein Sci 15:2558-2567

2. Fan B, Jiao D, Zhao X, Pang F, Xiao Q, Yu Z, Mao A, Guo R, Yuan W, Zhao P, He K, Li B (2017) Characterization of Chinese porcine epidemic diarrhea virus with novel insertions and deletions in genome. Sci Rep 7:44209

3. Hou Y, Lin CM, Yokoyama M, Yount BL, Marthaler D, Douglas AL, Ghimire S, Qin Y, Baric RS, Saif LJ, Wang Q (2017) Deletion of a 197-amino-acid region in the $\mathrm{N}$-terminal domain of spike protein attenuates porcine epidemic diarrhea virus in piglets. J Virol 91:e00227-e00227-17

4. Hulswit RJ, de Haan CA, Bosch BJ (2016) Coronavirus spike protein and tropism changes. Adv Virus Res 96:29-57

5. Kuo L, Godeke GJ, Raamsman MJ, Masters PS, Rottier PJ (2000) Retargeting of coronavirus by substitution of the spike glycoprotein ectodomain: crossing the host cell species barrier. J Virol 74:1393-1406

6. Lee C (2015) Porcine epidemic diarrhea virus: An emerging and re-emerging epizootic swine virus. Virol J 12:193

7. Lee DK, Cha SY, Lee C (2011) The N-terminal region of the porcine epidemic diarrhea virus spike protein is important for the receptor binding. Korean J Microbiol Biotechnol 39:140-145

8. Lee DK, Park CK, Kim SH, Lee C (2010) Heterogeneity in spike protein genes of porcine epidemic diarrhea viruses isolated in Korea. Virus Res 149:175-182

9. Lee S, Ko DH, Kwak WK, Lim CH, Moon SU, Lee DS, Lee C (2014) Reemergence of porcine epidemic diarrhea virus on Jeju Island. Korean J Vet Res 54:185-188

10. Lee S, Lee C (2014) Outbreak-related porcine epidemic diarrhea virus strains similar to US strains, South Korea, 2013. Emerg Infect Dis 20:1223-1226

11. Lee S, Lee C (2017) Complete genome sequence of a novel $\mathrm{S}$-insertion variant of porcine epidemic diarrhea virus from South Korea. Arch Virol 162:2919-2922

12. Lee S, Lee DU, Noh YH, Lee SC, Choi HW, Yang HS, Seol JH, Mun SH, Kang WM, Yoo H, Lee C (2019) Molecular characteristics and pathogenic assessment of porcine epidemic diarrhea virus 
isolates from the 2018 endemic outbreaks on Jeju Island, South Korea. Transb Emerg Dis. https://doi.org/10.1111/tbed.13219

13. Li C, Li W, de Esesarte EL, Guo H, van den Elzen P, Aarts E, van den Born E, Rottier PJ, Bosch BJ (2017) Cell attachment domains of the porcine epidemic diarrhea virus spike protein are key targets of neutralizing antibodies. J Virol 12:e00273-e00275

14. Lin CM, Annamalai T, Liu X, Gao X, Lu Z, El-Tholoth M, Hu H, Saif LJ, Wang Q (2015) Experimental infection of a US spikeinsertion deletion porcine epidemic diarrhea virus in conventional nursing piglets and cross-protection to the original US PEDV infection. Vet Res 46:134

15. Okda FA, Lawson S, Singrey A, Nelson J, Hain KS, Joshi LR, Christopher-Hennings J, Nelson EA, Diel DG (2017) The S2 glycoprotein subunit of porcine epidemic diarrhea virus contains immunodominant neutralizing epitopes. Virology 509:185-194
16. Suzuki T, Shibahara T, Yamaguchi R, Nakade K, Yamamoto T, Miyazaki A, Ohashi S (2016) Pig epidemic diarrhoea virus S gene variant with a large deletion non-lethal to colostrum-deprived newborn piglets. J Gen Virol 97:1823-1828

17. Van Diep N, Norimine J, Sueyoshi M, Lan NT, Yamaguchi R (2017) Novel porcine epidemic diarrhea virus (PEDV) variants with large deletions in the spike (S) gene coexist with PEDV strains possessing an intact $S$ Gene in domestic pigs in Japan: a new disease situation. PLoS One 12:e170126

Publisher's Note Springer Nature remains neutral with regard to jurisdictional claims in published maps and institutional affiliations. 